\title{
Development and validation of a new algorithm model for differential diagnosis between Crohn's disease and intestinal tuberculosis: a combination of laboratory, imaging and endoscopic characteristics
}

\author{
Yi Lu ${ }^{1,2}$, Yonghe Chen ${ }^{2,3}$, Xiang Peng ${ }^{2,4}$, Jiayin Yao ${ }^{2,4}$, Weijie Zhong ${ }^{1,2}$, Chujun $\mathrm{Li}^{1,2^{*}}$ and Min Zhi ${ }^{2,4^{*}}$
}

\begin{abstract}
Background: Sometimes in clinical practice, it is a great challenge to distinguish Crohn's disease (CD) and intestinal tuberculosis (ITB), we conducted this study to identify simple and useful algorithm for distinguishing them.

Methods: We retrospectively reviewed the medical history of the patients who were diagnosed as ITB or CD. We firstly identified ITB patients, and then the patients diagnosed with CD were matched by age, sex, and admission time in a 1:1 ratio. Patients who admitted between May 1,2013 and April 30, 2019 were regarded as training cohort, and patients admitted between May 1, 2019 and May 1, 2020 were regarded as validation cohort. We used multivariate analysis to identify the potential variables, and then we used $\mathrm{R}$ package rpart to build the classification and regression tree (CART), and validated the newly developed model.

Results: In total, the training cohort included 84 ITB and 84 CD patients, the validation cohort included 22 ITB and $22 \mathrm{CD}$ patients. Multivariate analysis showed that, positive interferon-gamma release assays (IGRAs), $\geq 4$ segments involved, longitudinal ulcer, circular ulcer, and aphthous ulcer were confirmed as independent discriminating factors. Using these parameters to build the CART model made an overall accuracy rate was $88.64 \%$, with sensitivity, specificity, NPV, and PPV being 90.91\%, 86.36\%, 90.48\% and 86.96\%, respectively.
\end{abstract}

Conclusion: We developed a simple and novel algorithm model covering laboratory, imaging, and endoscopy parameters with CART to differentiate ITB and CD with good accuracy. Positive IGRAs and circular ulcer were suggestive of ITB, while $\geq 4$ segments involved, longitudinal ulcer, and aphthous ulcer were suggestive of CD.

Keywords: Crohn's disease, Intestinal tuberculosis, Diagnosis, Algorithm, CART

*Correspondence: lichujun@mail.sysu.edu.cn; zhimin@mail.sysu.edu.cn 1 Department of Gastrointestinal Endoscopy, the Sixth Affiliated Hospital, Sun Yat-Sen University, Guangzhou 510655, People's Republic of China ${ }^{2}$ Guangdong Provincial Key Laboratory of Colorectal and Pelvic Floor Diseases, The Sixth Affiliated Hospital, Sun Yat-Sen University, 26 Yuancun Erheng Road, Guangzhou 510655, People's Republic of China

Full list of author information is available at the end of the article

\section{Introduction}

Crohn's disease (CD) and intestinal tuberculosis (ITB) are two intestinal diseases with different etiology, pathogenesis, and treatment. Sometimes in clinical practice, it is a great challenge to distinguish the two diseases. It was reported that the misdiagnosis rate between the two diseases could reach $50 \%-70 \%$ [1]. The diagnosis of ITB can be made when acid fast bacilli (AFB) and 
granulomas with caseous necrosis are found in histopathology, but the positive rates are low $[2,3]$. If ITB could not be excluded, 8-12 weeks of empirical antituberculosis treatment (ATT) is recommended, avoiding the severe adverse events if immunosuppressive drugs are prescribed to ITB patients by mistake $[4,5]$. Nevertheless, the ATT can delay CD treatment, and ATT has many side effects $[5,6]$. Hence, models that can be used at the bedside for easy and accurate differential diagnosis would be extremely useful in clinical practice. As a result, many researchers have tried to identify parameters and models that could improve the accuracy rate [7-9]. For laboratory parameters, interferon-gamma release assays (IGRAs, including T-SPOT and QuantiFERON-TB Gold) were important in differentiating ITB and $\mathrm{CD}$, and a positive result was more likely to be ITB [10]. For imaging characteristics, target sign, comb sign, adipose creeping sign and involvement of fewer than 4 segments were reported to be vital parameter, and the former three were more like to be the characteristics of $C D$, while the last was more likely to be ITB $[8,9,11-13]$. For endoscopic parameters, longitudinal ulcers, transverse ulcers, aphthous ulcers, cobblestone, and stricture were proved to be useful, and transverse ulcers were specific to ITB, while the others were more likely to be CD [14]. There parameters are of various kinds, and the scoring systems also vary from each other, and some models are complex to use. Therefore, we conducted this study to investigate the laboratory, imaging and endoscopic characteristics of $\mathrm{CD}$ and ITB, and to identify simple and useful algorithm for distinguishing the two diseases.

\section{Methods \\ Patients}

We retrospectively reviewed the medical history of the patients who were diagnosed as ITB or CD between May 1, 2013 and May 1, 2020. The inclusion criteria were: (1) had results of IGRAs; (2) had undergone colonoscopy or trans-anal enteroscopy in our hospital so as to obtain the endoscopic characteristics; (3) had the results of computed tomography enterography (CTE) or magnetic resonance enterography (MRE) so as to obtain imaging characteristics; (4) the diagnosis was not confirmed when the patients visited our hospital; (5) the patients had not received any treatment or had received ATT or glucocorticoids or immunosuppressive agents for no more than 1 week; (6) the diagnosis of either ITB or CD could be confirmed. The exclusion criteria were: (1) the result of IGRAs or endoscopic characters or CTE/MRE was not complete; (2) had received treatments for more than 1 week; (3) final diagnosis could not be confirmed; (4) ITB and CD both existed; (5) had colectomy before this hospitalization.

\section{Diagnostic criteria}

The confirmed diagnosis for ITB required one of the following: (a) caseating granulomas detected in endoscopy biopsy, surgical specimen, or mesenteric lymph nodes; (b) demonstration of AFB on smears or histological sections or positive culture for AFB; (c) strong suspicion of ITB with a good response to ATT without recurrence; a good response to ATT was determined by relief of symptoms and disappearance of ulcerations on endoscopic examination for at least 6 months of follow-up [7, 8, 15].

The confirmed diagnosis for $\mathrm{CD}$ was made based on the management consensus of inflammatory bowel disease for the Asia-Pacific region [16], which included clinical, endoscopic, histological, and radiological characteristics and/or biochemical evaluation.

\section{Study design}

Firstly, we identified the patients diagnosed with ITB who met all the inclusion criteria (group ITB). Then the patients diagnosed with $\mathrm{CD}$ were matched by age, sex, and admission time in a 1:1 ratio (group CD). We reviewed the medical history of the patients including age, sex, weight, height, symptoms, present and past history, laboratory results [blood routine, erythrocyte sedimentation rate (ESR), albumin, hsCRP, stool routine, IGRAs and PPD skin test], endoscopy results (location of lesions, shape of ulcers, cobblestone appearance, pseudopolyp, scar, and stricture), imaging results (enlargement of celiac lymph nodes, peritoneal abscess, bowel wall thickening, stricture, comb sign, target sign, adipose creeping sign [13], $\geq 4$ segments involved, intestinal fistula, perianal diseases and chest X-ray/CT), pathology results (acid fast bacilli staining, granulomas, caseous necrosis or non-caseous necrosis), diagnosis, treatment and follow-up results. The definitions of each laboratory, endoscopic and imaging finding are presented in the Additional file 1 [11-13, 17, 18]. Patients who admitted between May 1, 2013 and April 30, 2019 were regarded as training cohort, the information of which was used to develop the algorithm; and patients admitted between May 1, 2019 and May 1, 2020 were regarded as validation cohort, the information of which was used to validate the algorithm. The study was approved by the ethics committee in our hospital.

\section{Statistical analysis}

IBM SPSS Statistic Version 24.0.0.0 was used to compare the differences between the two groups. Continuous variables without normal distribution were presented with median (lower and upper interquartile range), and tested by nonparametric Wilcoxon rank-sum test. Categorical variables were presented with number (percentage), and 
were tested by $x^{2}$ test. Statistical significance was defined as $P<0.05$ (two-tailed). After the comparison, we identified some parameters which had statistical differences between the two groups, and selected them as potential variables to build the algorithm.

The study end point was to develop an algorithm to differentiate ITB and CD, and to validate it. One hundred and sixty-eight and forty-four cases were allocated to the training cohort and validation cohort, respectively. Parameters with statistical significance were enrolled into logistic regression for multivariate analysis. Independent discriminating factors selected by the logistic model were then used to build a decision tree based on the R package rpart (https://CRAN.R-project.org/package $=$ rpart). Briefly, this is a nonparametric regression method based on binary recursive partitioning of data in the training set to build a model like a tree structure, which is also called classification and regression tree (CART). It incrementally divides the data into smaller subclasses according to the amount of information gained by each partition into a subclass until no additional information could be gained by a split [19]. It generated a tree structure flow chart to differentiate ITB and CD that is easy to interpret. The overall sensitivity, specificity, negative predictive value (NPV), positive predictive value (PPV) and predictive accuracy of the CART were evaluated using data of the validation cohort. $P<0.05$ was regarded as statistical significance. $\mathrm{R}$ version 4.0.2 (R Foundation for Statistical Computing, Vienna, Austria. https://www.R-project.org) was used to perform the statistical analysis.

\section{Results}

\section{Baseline and laboratory characteristics of the training cohort}

In total, the training cohort included 84 ITB and 84 CD patients, the validation cohort included 22 ITB and 22 CD patients. In the training cohort, 31 patients $(36.90 \%)$ have received empirical ATT in group ITB; the ratio of patients with diarrhea, symptoms of perianal diseases (perianal pain, perianal discomfort, perianal pus or fluids), anemia, PLT $>300 * 10^{\wedge} 9 / \mathrm{L}$, hsCRP $>3 \mathrm{mg} / \mathrm{L}$, and positive occult blood test was statistically higher in group CD than that in group ITB; the ratio of positive PPD skin test and positive IGRAs was higher in group ITB (The details are shown in Table 1).

\section{Imaging characteristics of the training cohort}

The CTE/MRE results analysis showed that, the ratio of patients with comb sign, target sign, adipose creeping sign and $\geq 4$ segments involved was significantly higher in group CD than that in group ITB. Anal magnetic resonance image (MRI) results showed that more patients in group CD have anal fistula or perianal abscess. Chest
Table 1 Clinical and laboratory characteristics of the training cohort

\begin{tabular}{|c|c|c|c|}
\hline & ITB $(n=84)$ & $C D(n=84)$ & $P$ value \\
\hline Age, years & $32(24-46)$ & $32(26-46)$ & 0.685 \\
\hline Male, n (\%) & $52(61.90)$ & $52(61.90)$ & 1 \\
\hline \multicolumn{4}{|l|}{ Symptoms } \\
\hline Abdominal pain & $33(39.29)$ & $32(38.10)$ & 0.874 \\
\hline Diarrhea & $8(9.52)$ & $33(39.29)$ & $<0.0001$ \\
\hline Gastrointestinal bleeding & $8(9.52)$ & $11(13.10)$ & 0.465 \\
\hline Weight loss & $33(39.29)$ & $25(29.76)$ & 0.194 \\
\hline Fever & $5(5.95)$ & $2(2.38)$ & 0.443 \\
\hline Perianal diseases & $11(13.10)$ & $33(39.29)$ & $<0.0001$ \\
\hline History of appendicectomy & $6(7.14)$ & $4(4.76)$ & 0.514 \\
\hline Anemia & $21(25)$ & $33(39.29)$ & 0.047 \\
\hline $\mathrm{PLT}>300^{*} 10 \wedge 9 / \mathrm{L}$ & $29(34.52)$ & $47(55.95)$ & 0.005 \\
\hline $\mathrm{WBC}>10^{*} 10 \wedge 9 / \mathrm{L}$ & $10(11.90)$ & $12(14.29)$ & 0.647 \\
\hline$E S R>20 \mathrm{~mm} / \mathrm{h}^{\#}$ & $52(61.90)$ & $55(66.27)$ & 0.557 \\
\hline Hypoalbuminemia & $19(22.62)$ & $29(34.52)$ & 0.088 \\
\hline $\mathrm{hsCRP}>3 \mathrm{mg} / \mathrm{L}^{\#}$ & $42(50.60)$ & $65(78.31)$ & $<0.0001$ \\
\hline Positive occult blood test ${ }^{\#}$ & $18(21.69)$ & $29(36.25)$ & 0.04 \\
\hline Positive PPD skin test ${ }^{\#}$ & $57(68.67)$ & $9(12.5)$ & $<0.0001$ \\
\hline Positive IGRAs & $76(90.48)$ & $12(14.29)$ & $<0.0001$ \\
\hline
\end{tabular}

ITB, intestinal tuberculosis; CD, Crohn's disease; PLT, platelet; WBC, white blood cell; ESR, erythrocyte sedimentation rate

\# Not all patients had the result

$\mathrm{X}$-ray/CT results showed that more patients in group ITB had pulmonary TB and calcification (The imaging characteristics of the training cohort are shown in Table 2).

\section{Endoscopy and pathology characteristics of the training cohort}

The endoscopy results showed that, the ratio of patients with longitudinal ulcer, aphthous ulcer (the endoscopy images are shown in Fig. 1), rectum involvement, and sigmoid colon involvement was significantly higher in group CD than that in group ITB. More patients in group ITB had circular ulcer, and the pathology result showed that, the ratio of caseous necrosis was also higher in group ITB (The endoscopy and pathology characteristics of the training cohort are shown in Table 3).

\section{Multivariate analysis and diagnostic values of the factors}

When these parameters were entered into a multivariate analysis, positive IGRAs, $\geq 4$ segments involved, longitudinal ulcer, circular ulcer, and aphthous ulcer were confirmed as independent discriminating factors (Table 4 shows the multivariate analysis results). Further analysis of the diagnostic values of these factors is shown in Table 5. The most sensitive and specific parameters for differentiating ITB and CD when used 
Table 2 Imaging characteristics of the training cohort

\begin{tabular}{|c|c|c|c|}
\hline & ITB $(n=84)$ & $C D(n=84)$ & $P$ value \\
\hline \multicolumn{4}{|l|}{ CTE/MRE, n (\%) } \\
\hline $\begin{array}{l}\text { Enlargement of celiac lymph } \\
\text { nodes }\end{array}$ & $30(35.71)$ & $25(29.76)$ & 0.411 \\
\hline Peritoneal abscess & $2(2.38)$ & $7(8.33)$ & 0.087 \\
\hline Bowel wall thickening & $81(96.43)$ & $82(97.62)$ & 0.65 \\
\hline Stricture & $19(22.62)$ & $29(34.52)$ & 0.088 \\
\hline Comb sign & $15(17.86)$ & $60(71.43)$ & $<0.0001$ \\
\hline Target sign & $29(34.52)$ & $62(73.81)$ & $<0.0001$ \\
\hline Adipose creeping sign & $7(8.33)$ & $31(36.9)$ & $<0.0001$ \\
\hline$\geq 4$ segments involved & $15(17.86)$ & $68(80.95)$ & $<0.0001$ \\
\hline Intestinal fistula & $2(2.38)$ & $6(7.14)$ & 0.147 \\
\hline \multicolumn{4}{|l|}{ Anal MRI } \\
\hline Anal fistula or perianal abscess & 13 (39.39) & $52(86.67)$ & $<0.0001$ \\
\hline \multicolumn{4}{|l|}{ Chest $X$-ray/CT } \\
\hline Pulmonary TB & $34(40.48)$ & $0(0)$ & $<0.0001$ \\
\hline Fibrosis & $22(26.19)$ & $14(16.67)$ & 0.133 \\
\hline Calcification & $12(14.29)$ & $4(4.76)$ & 0.035 \\
\hline Infection ${ }^{*}$ & $7(8.33)$ & $3(3.57)$ & 0.192 \\
\hline
\end{tabular}

ITB, intestinal tuberculosis; CD, Crohn's disease; CTE, computed tomography enterography; MRE, magnetic resonance enterography; MRI, magnetic resonance image; $\mathrm{CT}$, computed tomography; $\mathrm{TB}$, tuberculosis

" Infection but not sufficient to support TB infection;" not all patients had the result

in isolation were circular ulcer (sensitivity 100\%) and longitudinal ulcer (specificity 100\%). However, circular ulcer was not specific for ITB, because $50 \%$ of CD patients had a circular ulcer. Similarly, longitudinal ulcer was specific to $C D$, but the true positive rate was only $40.91 \%$ in CD patients.

\section{The CART analysis of training and validation cohorts}

The first factor that split the training population was IGRAs. $86.36 \%$ of the patients with positive IGRAs were diagnosed as ITB, while $90 \%$ of the patients with negative IGRAs were diagnosed as CD. The CART analysis result for the training cohort is shown in Fig. 2a. From the figure, we could find that, patients with positive IGRAs and circular ulcer but without aphthous ulcer have high reliability in the diagnosis of ITB; patients with negative IGRAs and with $\geq 4$ segments involved/ longitudinal ulcer have high reliability in the diagnosis of CD.

When we tested this algorithm using the data in the validation cohort, the overall accuracy rate was $88.64 \%$ (39 in 44 patients). The CART analysis in the validation cohort is shown in Fig. 2b. The sensitivity, specificity, NPV, and PPV of differential diagnosis of ITB from CD was $90.91 \%, 86.36 \%, 90.48 \%$ and $86.96 \%$, respectively (Table 6).

\section{Discussion}

Differentiating ITB from CD is sometimes very difficult in some patients, especially in the areas where both ITB and CD are prevalent, as there are many similarities between ITB and CD in clinical manifestations, laboratory results, imaging, endoscopic and even histological characteristics. On the other hand, the misdiagnosis or delayed diagnosis can result in tubercle bacillus diffusion, drug adverse events and medical expenditure increase [20]. Hence, there is an urgent need for developing accurate and easy diagnostic tools to differentiate ITB and $\mathrm{CD}$. Many previous studies have been conducted to construct models that could improve the accuracy $[7-9,11$, $21]$, but they used different diagnostic models and scoring systems, and some of them were not easy to use, as they may need a calculator or a computer. In this study, we finally selected five parameters (positive IGRAs, $\geq 4$ segments involved, longitudinal ulcer, circular ulcer, and aphthous ulcer) covering laboratory, imaging and endoscopic characteristics, and constructed the CART algorithm, which could be used at the bedside for easy and accurate differential. To our knowledge, this is the first study to use CART to differentiate ITB and CD.

In this study, we firstly compared the clinical, imaging, endoscopic and pathologic characteristics between ITB and $\mathrm{CD}$ in the training cohort. For clinical characteristics in the training cohort, patients with CD were more likely to have diarrhea, and symptoms of perianal diseases, this was in accordance with previous studies [7, 21]. For laboratory characteristics, patients with $\mathrm{CD}$ were more likely to have anemia, PLT $>300^{*} 10^{\wedge} 9 / \mathrm{L}$, hsCRP $>3 \mathrm{mg} / \mathrm{L}$, and positive occult blood test than ITB, the results were close to some of the previous studies, but some were not in consistency [7, 9, 21]. Patients with ITB had much higher rate of positive IGRAs, which was a very important parameter in the CART, as it split the patients in the first step. A meta-analysis showed that, the pooled sensitivity and specificity of IGRAs for the differential diagnosis of ITB from CD were $82.8 \%$ and $86.7 \%$, respectively, indicating that it was a good independent discriminating factor [10]. In this study and in clinical, some patients had a positive TB test but the final diagnosis was CD, the algorithm helped us to differentiate this as follows: if they also had aphthous ulcer, then the probability of $C D$ was about $83.33 \%$; if they did not have aphthous ulcer no circular ulcer, but have $\geq 4$ segments involved and longitudinal ulcer, then probability of $\mathrm{CD}$ was very high, otherwise, the diagnosis would incline to ITB.

For imaging characteristics, especially CTE/MRE, patients with $\mathrm{CD}$ were more likely to show comb sign, target sign, adipose creeping sign and $\geq 4$ segments involved. Kedia et al. showed that comb sign was more common in CD [11], and no studies had compared target 


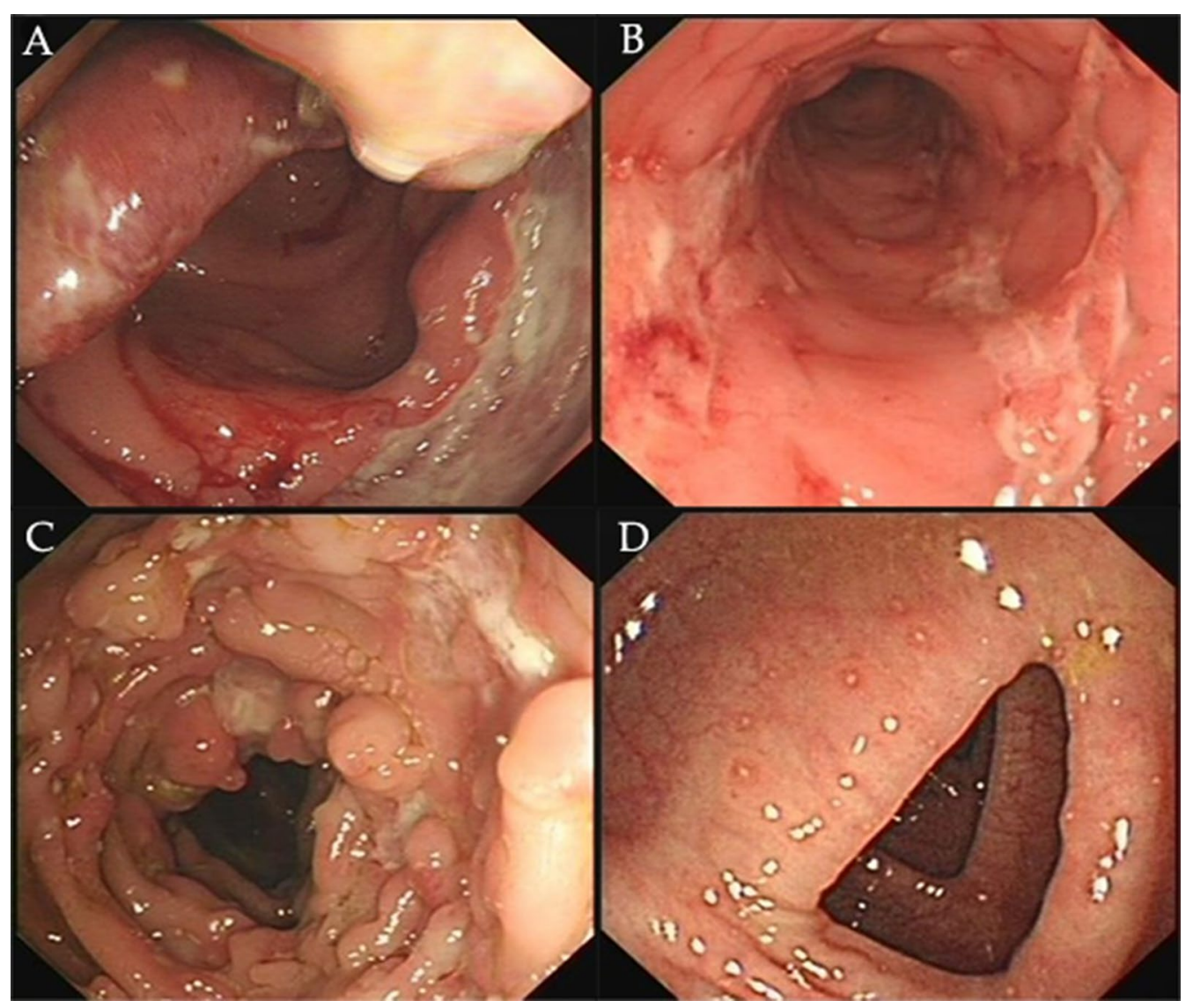

Fig. 1 Endoscopy images of a circular ulcer; $\mathbf{b}$ longitudinal ulcer; c cobblestone appearance; $\mathbf{d}$ aphthous ulcer

sign or adipose creeping sign in ITB and CD, but some studies have used them to build the differential models $[8,12]$. In our study, these three factors were probably easy to get influenced by other factors, and they failed to be selected as potential parameters in CART. Except for these signs in CTE, Kedia et al. also evaluated other characteristics in CTE, and they found that, necrotic lymph nodes were specific for ITB, while the combination of long segment lesion and visceral to subcutaneous fat ratio $(\mathrm{VF} / \mathrm{SC})$ ratio $>0.63$ was specific for $\mathrm{CD}$, using these characters could make an accuracy rate of $43 \%[11]$.

For endoscopic characteristics, rectum or sigmoid colon involvement and longitudinal ulcers, aphthous ulcers were more common in $\mathrm{CD}$, whereas transverse ulcers were more common in ITB, our results is the same as the previous published ones $[9,14,21]$. Lee et al. selected eight endoscopic characteristics along to build the models: transverse ulcers, less than 4 segment lesions, patulous ileocecal valve, and pseudopolyp (favored ITB); anorectal lesions, aphthous ulcers, longitudinal ulcers, and cobblestone appearance (favored $\mathrm{CD}$ ), and they hypothesized that if there were more parameters favored of ITB than CD, then a diagnosis of ITB could be made, and vice versa. This endoscopic model could make an accuracy rate of $87.5 \%$ and the PPV for CD and ITB was $95 \%$ and, respectively, indicating that a systematic analysis of endoscopic findings is very useful in differentiating ITB and CD [22]. In our study, we found that the shapes of the ulcers were very important parameters in differentiating ITB and CD, and transverse ulcers, longitudinal ulcers, and aphthous ulcers were all selected in our CART model. Regarding the importance of the shapes of the ulcers, we suppose that it may be possible to use the artificial intelligence (AI) technique, which is now very popular, to help in differentiating ITB and CD. As it can be taught to identify the characteristics of the ulcers (such as the shapes), and if we further enter some other parameters, after calculation and combination, the AI might give us a satisfied answer.

For pathologic characteristics, granulomas and noncaseous necrosis could be seen in both groups, while caseous necrosis and positive AFB staining was exclusive to ITB, but the sensitivity was low. Qian et al. compared granulomatous lymphangitis and granulomas in $\mathrm{CD}$ and 
Table 3 Endoscopy and pathology characteristics of the training cohort

\begin{tabular}{llll}
\hline & ITB $(\mathbf{n}=\mathbf{8 4})$ & $\mathbf{C D}(\mathbf{n}=\mathbf{8 4})$ & P value \\
\hline Endoscopy, $n$ (\%) & & & \\
Irregular ulcer & $56(66.67)$ & $71(60.71)$ & 0.422 \\
Longitudinal ulcer & $8(9.52)$ & $30(35.71)$ & $<0.0001$ \\
Circular ulcer & $26(30.95)$ & $1(1.19)$ & $<0.0001$ \\
Aphthous ulcer & $2(2.38)$ & $35(41.67)$ & $<0.0001$ \\
Cobblestone appearance & $4(4.76)$ & $11(13.10)$ & 0.058 \\
Pseudopolyp & $35(41.67)$ & $32(38.10)$ & 0.636 \\
Scar & $8(9.52)$ & $12(14.29)$ & 0.341 \\
Stricture & $24(28.57)$ & $19(22.62)$ & 0.377 \\
Fistula & $1(1.19)$ & $2(2.38)$ & 1 \\
Rectum involvement & $11(13.10)$ & $33(39.29)$ & $<0.0001$ \\
Sigmoid colon involvement & $11(13.10)$ & $31(36.90)$ & $<0.0001$ \\
Pathology & & & \\
Granulomas & $62(73.81)$ & $52(61.90)$ & 0.099 \\
Caseous necrosis & $8(9.52)$ & $0(0)$ & 0.007 \\
Non-caseous necrosis & $56(66.67)$ & $64(76.19)$ & 0.172 \\
Positive AFB staining & $4(16)$ & $0(0)$ & 1 \\
\hline
\end{tabular}

ITB, intestinal tuberculosis; CD, Crohn's disease; AFB, acid fast bacilli

\# Not all patients had the result

Table 4 Multivariate analysis to identify the independent discriminating factors

\begin{tabular}{|c|c|c|c|c|}
\hline Factors & Estimate & Standard error & $Z$ value & $P$ value \\
\hline Intercept & -0.05 & 0.64 & -0.083 & 0.934 \\
\hline Positive IGRAs & -3.62 & 0.77 & -4.672 & $<0.0001$ \\
\hline $\begin{array}{l}\geq 4 \text { segments } \\
\text { involved }\end{array}$ & 2.87 & 0.82 & 3.499 & 0.0005 \\
\hline Longitudinal ulcer & 2.06 & 0.93 & 2.225 & 0.026 \\
\hline Circular ulcer & -2.52 & 1.25 & -2.022 & 0.043 \\
\hline Aphthous ulcer & 2.52 & 1.21 & 2.089 & 0.037 \\
\hline $\begin{array}{l}\text { Comb sign/ target } \\
\text { sign/ Adipose } \\
\text { creeping sign }\end{array}$ & 0.53 & 0.72 & 0.742 & 0.458 \\
\hline Rectum involvement & -0.31 & 0.94 & -0.325 & 0.745 \\
\hline
\end{tabular}

Table 5 Accuracy of using each single discriminating factor in differentiating ITB and CD

\begin{tabular}{lllll}
\hline Factors & Sensitivity, \% & Specificity, \% & PPV, \% & NPV, \% \\
\hline Positive IGRAs & 86.36 & 82.82 & 82.61 & 85.71 \\
$\begin{array}{l}\geq 4 \text { segments } \\
\text { involved }\end{array}$ & 81.82 & 77.27 & 78.26 & 80.95 \\
$\begin{array}{l}\text { Longitudinal ulcer } \\
\text { Circular ulcer }\end{array}$ & 40.91 & 100 & 100 & 62.86 \\
Aphthous ulcer & 100 & 50 & 66.67 & 100 \\
\hline
\end{tabular}

$\mathrm{ITB}$, intestinal tuberculosis; CD, Crohn's disease; PPV, positive predictive value; $\mathrm{NPV}$, negative predictive value
ITB, and they concluded that, neither granulomatous lymphangitis nor granulomas was specific for $C D$, but their morphology and distribution could help in the differential diagnosis [23]. In our study, the pathologic characteristics were not selected as parameters to build the CART model, but we suppose that, if we study the morphology and distribution of granulomas next time, we may find some meaningful parameters.

Using a single parameter to distinguishing ITB and $\mathrm{CD}$ has very limited use in clinical practice, as its sensitivity or specificity is usually low. Hence, an integration of various categories of valuable parameters to establish a model could possibly help to deal with this question. Mao et al. selected 2 CTE findings and 8 endoscopic findings to build the model, and they found that the accuracy rate increased from 71.6 to $88.3 \%$, compared with using endoscopic findings alone [24]. Bae et al. also developed a scoring system using 8 endoscopic findings, chest ray, small bowel follow through, and 2 laboratory tests [anti-Saccharomyces cerevisiae antibodies (ASCA) and IGRAs], which showed very good results, with an accuracy rate of $96.3 \%$ [9]. But there is limitation in their model, as in our hospital and many other hospitals in China, ASCA is not routine tested, we may fail in using this model. Some studies used likelihood ratio models to differentiate these two diseases [7, 25], and their accuracy rate could reach $95.7 \%$. And there were many other models used as Limsrivilai et al. showed in their systematic review [5], but these models usually contained a very complex formula, and need a calculator, so they are difficult to be applied at the bedside. CART can dissect complicated clinical situations and identifies homogeneous patient groups, so it is able to generate clinical decision trees. In our CART model, the parameters are easy to obtain, and does not need any calculation, we think it will be more convenient to use. As in the previous models, they usually used logistic regression or nomogram which calculated a probability of disease, were inconvenient for clinicians, as they generally think in terms of categories, such as "yes" or "no", rather than think about probability. So the CART model is ideally suited to generation of decision trees for the clinicians.

There are some limitations in this study. Firstly, this is a single-centered, retrospective study, and the sample size is limited, and due to its retrospective, some other factors could not be assessed, on the other hand, though we validated this algorithm, it was still internal validation, so prospective, larger sample-sized, and multi-centered study is still needed to make it more convincible and can be applied to other centers. Secondly, the patients with $\mathrm{CD}$ were matched by age, sex, and admission time in a 1:1 ratio, while the former two elements might be important parameters in other studies $[8,21]$, while we did not 


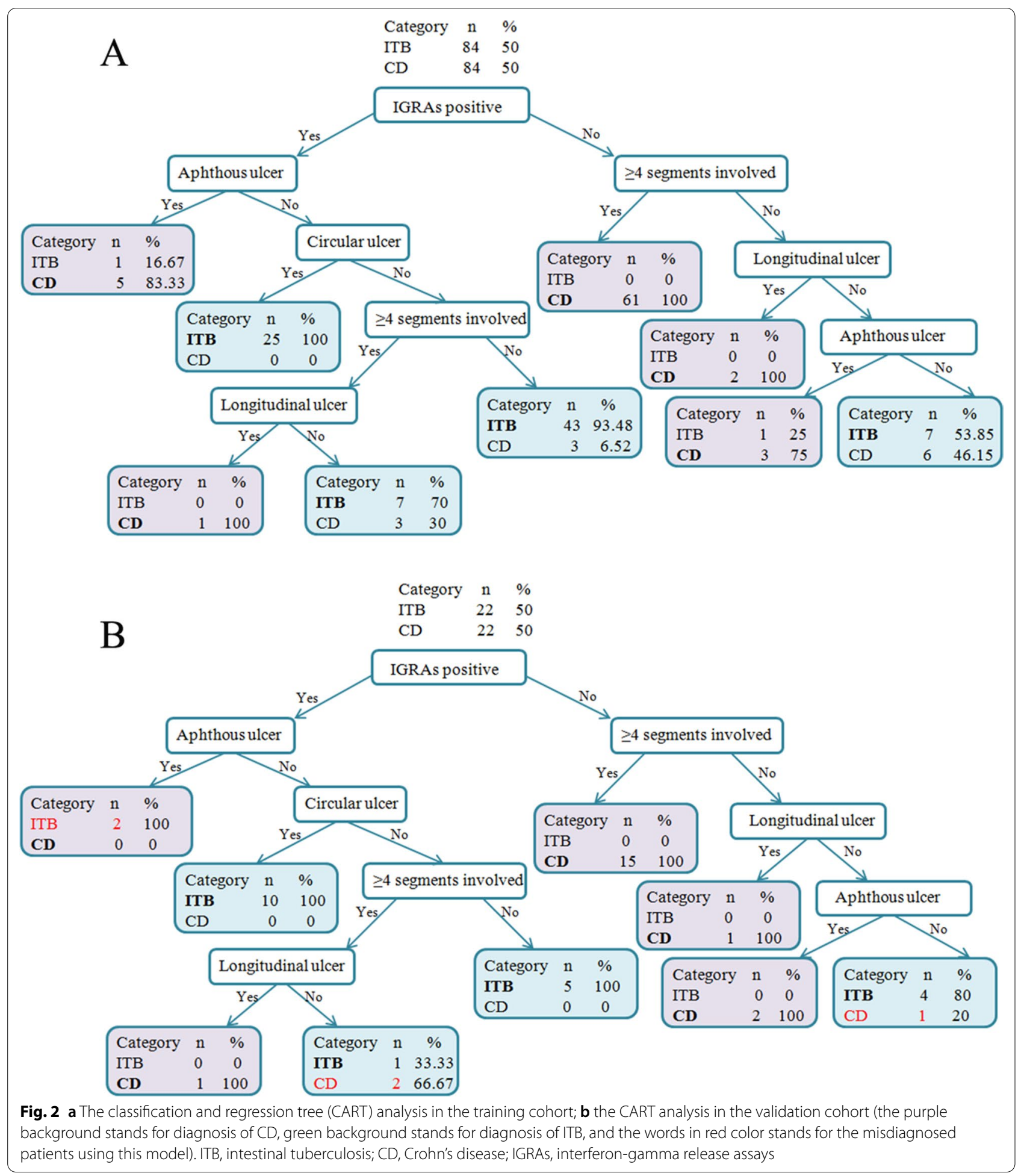

include these parameters, as we planned to build the CART, and the variables used to build the model were categorical variables, as age was not categorical variable, and we cannot find a cut-off point younger than which was $\mathrm{CD}$, so age was not used to enter the model. As for gender, we thought it was impossible to differentiate the two diseases based on gender in the CART model, so we matched the patients of the same age and gender, 
Table 6 Differential diagnosis based on the classification and regression tree in validation cohort

\begin{tabular}{llll}
\hline $\begin{array}{llll}\text { Predicted category } \\
\text { (n) }\end{array}$ & Actual category & Total \\
\cline { 2 - 3 } & ITB $(\mathbf{n}=\mathbf{2 2})$ & $\mathbf{C D}(\mathbf{n}=\mathbf{2 2})$ & \\
\hline ITB & 20 & 3 & 23 \\
CD & 2 & 19 & 21 \\
Total & 22 & 22 & 44 \\
\hline
\end{tabular}

ITB, intestinal tuberculosis; $C D$, Crohn's disease

and focused more on other variables we were interested in. Thirdly, the diagnostic algorithm started with IGRAs result, while in clinical practice, sometimes we might have indeterminate result, than it was not suitable to use this model. Actually, this usually occurs in immunosuppressed patients, and the incidence was reported to be $0 \%$ to $9.7 \%$ [26-28], though not high, it was really a thorny problem in clinical. In the future work, we must investigate how to deal with patients with indeterminate result. Fourthly, CART itself also has disadvantages, for example, in some branches, the accuracy rate was not that satisfied, and that as CART is not based upon a probabilistic model, so the CART tree cannot provide the probability level or confidence interval of the predictions $[29,30]$.

In conclusion, we developed a simple and novel algorithm model covering laboratory, imaging, and endoscopy parameters with CART to differentiate ITB and CD with good accuracy, but external validation is warranted. Except for our model, many models have been proposed in the previous published studies, and we can select the model that is most appropriate and easy to apply.

\footnotetext{
Abbreviations

CD: Crohn's disease; ITB: Intestinal tuberculosis; CART: Classification and regression tree; AFB: Acid fast bacilli; ATT: Antituberculosis treatment; CTE: Computed tomography enterography; MRE: Magnetic resonance enterography; ESR: Erythrocyte sedimentation rate; NPV: Negative predictive value; PPV: Positive predictive value; Al: Artificial intelligence; ASCA: Anti-Saccharomyces cerevisiae antibodies.
}

\section{Supplementary Information}

The online version contains supplementary material available at https://doi. org/10.1186/s12876-021-01838-x.

Additional file 1. Definitions of the Laboratory, Imaging and Endoscopic Findings.

\section{Acknowledgements}

Not applicable.

\section{Authors' contributions}

LY collected the data, performed the statistical analysis and wrote the manuscript draft. CYH collected the data, performed the statistical analysis. PX, YJY and ZWJ collected the data. ZM and LCJ designed the study and revised the manuscript.
Funding

None.

Availability of data and materials

The datasets used are available from the corresponding author on reasonable request.

\section{Competing interests}

The authors declare that they have no competing interests.

\section{Consent for publication}

Not applicable.

\section{Ethics approval}

This study was approved by the ethical principles of the Declaration of Helsinki 1964 and the ethics committee of the Sixth Affiliated Hospital, Sun Yat-sen University, and the informed consent was waived.

\section{Author details}

${ }^{1}$ Department of Gastrointestinal Endoscopy, the Sixth Affiliated Hospital, Sun Yat-Sen University, Guangzhou 510655, People's Republic of China. ${ }^{2}$ Guangdong Provincial Key Laboratory of Colorectal and Pelvic Floor Diseases, The Sixth Affiliated Hospital, Sun Yat-Sen University, 26 Yuancun Erheng Road, Guangzhou 510655, People's Republic of China. ${ }^{3}$ Department of Gastrointestinal Surgery, the Sixth Affiliated Hospital, Sun Yat-Sen University, Guangzhou 510655, People's Republic of China. ${ }^{4}$ Department of Gastroenterology, the Sixth Affiliated Hospital, Sun Yat-Sen University, Guangzhou 510655, People's Republic of China.

Received: 28 January 2021 Accepted: 31 May 2021

Published online: 13 July 2021

\section{References}

1. Ng SC, Hirai HW, Tsoi KK, et al. Systematic review with meta-analysis: accuracy of interferon-gamma releasing assay and anti-Saccharomyces cerevisiae antibody in differentiating intestinal tuberculosis from Crohn's disease in Asians. J Gastroenterol Hepatol. 2014;29(9):1664-70.

2. Pulimood AB, Peter S, Ramakrishna B, et al. Segmental colonoscopic biopsies in the differentiation of ileocolic tuberculosis from Crohn's disease. J Gastroenterol Hepatol. 2005;20(5):688-96.

3. Almadi MA, Ghosh S, Aljebreen AM. Differentiating intestinal tuberculosis from Crohn's disease: a diagnostic challenge. Am J Gastroenterol. 2009;104(4):1003-12.

4. Ooi CJ, Makharia GK, Hilmi I, et al. Asia Pacific Consensus Statements on Crohn's disease. Part 1: Definition, diagnosis, and epidemiology: (Asia Pacific Crohn's Disease Consensus-Part 1). J Gastroenterol Hepatol. 2016;31(1):45-55

5. Limsrivilai J and N Pausawasdi, Intestinal tuberculosis or Crohn's disease: a review of the diagnostic models designed to differentiate between these two gastrointestinal diseases. Intest Res, 2020.

6. Tandon HD, Prakash A. Pathology of intestinal tuberculosis and its distinction from Crohn's disease. Gut. 1972;13(4):260-9.

7. Wu X, Huang H, Hou H, et al. Diagnostic Performance of a 5-Marker Predictive Model for Differential Diagnosis Between Intestinal Tuberculosis and Crohn's Disease. Inflamm Bowel Dis. 2018:24(11):2452-60.

8. He Y, Zhu Z, Chen Y, et al. Development and Validation of a Novel Diagnostic Nomogram to Differentiate Between Intestinal Tuberculosis and Crohn's Disease: A 6-year Prospective Multicenter Study. Am J Gastroenterol. 2019;114(3):490-9.

9. Bae JH, Park SH, Ye BD, et al. Development and Validation of a Novel Prediction Model for Differential Diagnosis Between Crohn's Disease and Intestinal Tuberculosis. Inflamm Bowel Dis. 2017:23(9):1614-23.

10. Xu H, Li Y, Qian JM. A Meta-analysis of the accuracy of interferon- $\gamma$ release assays in differentiating intestinal tuberculosis from Crohn's disease in Asia. Zhonghua Nei Ke Za Zhi. 2016;55(7):535-40.

11. Kedia S, Madhusudhan KS, Sharma R, et al. Combination of increased visceral fat and long segment involvement: Development and validation of an updated imaging marker for differentiating Crohn's disease from intestinal tuberculosis. J Gastroenterol Hepatol. 2018;33(6):1234-41. 
12. Huang $X$, Liao WD, Yu C, et al. Differences in clinical features of Crohn's disease and intestinal tuberculosis. World J Gastroenterol. 2015;21(12):3650-6.

13. Golder WA. The "creeping fat sign"-really diagnostic for Crohn's disease? Int J Colorectal Dis. 2009;24(1):1-4.

14. Kedia S, Das P, Madhusudhan KS, et al. Differentiating Crohn's disease from intestinal tuberculosis. World J Gastroenterol. 2019;25(4):418-32.

15. Patel N, Amarapurkar D, Agal S, et al. Gastrointestinal luminal tuberculosis: establishing the diagnosis. J Gastroenterol Hepatol. 2004;19(11):1240-6.

16. Ouyang Q, Tandon R, Goh KL, et al. Management consensus of inflammatory bowel disease for the Asia-Pacific region. J Gastroenterol Hepatol. 2006;21(12):1772-82.

17. Feng, PJ, Y Wu and CS Ho, et al., Impact of T-Cell Xtend on T-SPOT.TB Assay in High-Risk Individuals after Delayed Blood Sample Processing. J Clin Microbiol, 2021.

18. Li J, Li P, Bai J, et al. Discriminating potential of extraintestinal systemic manifestations and colonoscopic features in Chinese patients with intestinal Behcet's disease and Crohn's disease. Chin Med J (Engl). 2015;128(2):233-8.

19. Segal MR, Bloch DA. A comparison of estimated proportional hazards models and regression trees. Stat Med. 1989;8(5):539-50.

20. Makharia GK, Srivastava S, Das P, et al. Clinical, endoscopic, and histological differentiations between Crohn's disease and intestinal tuberculosis. Am J Gastroenterol. 2010;105(3):642-51.

21. Jung Y, Hwangbo Y, Yoon SM, et al. Predictive Factors for Differentiating Between Crohn's Disease and Intestinal Tuberculosis in Koreans. Am J Gastroenterol. 2016:111(8):1156-64.

22. Lee YJ, Yang SK, Byeon JS, et al. Analysis of colonoscopic findings in the differential diagnosis between intestinal tuberculosis and Crohn's disease. Endoscopy. 2006;38(6):592-7.
23. Qian Y, He W, Zheng CY, et al. Significance of granuloma and granulomatous lymphangitis in the differential diagnosis of Crohn's disease. J Dig Dis. 2020;21(8):454-61.

24. Mao R, Liao WD, He Y, et al. Computed tomographic enterography adds value to colonoscopy in differentiating Crohn's disease from intestinal tuberculosis: a potential diagnostic algorithm. Endoscopy. 2015;47(4):322-9.

25. Zhao XS, Wang ZT, Wu ZY, et al. Differentiation of Crohn's disease from intestinal tuberculosis by clinical and CT enterographic models. Inflamm Bowel Dis. 2014;20(5):916-25.

26. Meier NR, Volken T, Geiger M, et al. Risk Factors for Indeterminate Interferon-Gamma Release Assay for the Diagnosis of Tuberculosis in Children-A Systematic Review and Meta-Analysis. Front Pediatr. 2019;7:208.

27. Shangguan $Y$, Fang H, Wang S, et al. Risk factors for negative T-SPOT.TB assay results in patients with confirmed active tuberculosis: A retrospective study. J Infect Dev Ctries. 2020;14(11):1288-95.

28. Nozawa T, Mori M, Nishimura K, et al. Usefulness of two interferon- $\gamma$ release assays for rheumatic disease. Pediatr Int. 2016:58(5):347-52.

29. Lee SK, Kim BK, Kim TI, Kim WH. Differential diagnosis of intestinal Behçet's disease and Crohn's disease by colonoscopic findings. Endoscopy. 2009;41(1):9-16.

30. Costanza MC, Paccaud F. Binary classification of dyslipidemia from the waist-to-hip ratio and body mass index: a comparison of linear, logistic, and CART models. BMC Med Res Methodol. 2004;4:7.

\section{Publisher's Note}

Springer Nature remains neutral with regard to jurisdictional claims in published maps and institutional affiliations.
Ready to submit your research? Choose BMC and benefit from:

- fast, convenient online submission

- thorough peer review by experienced researchers in your field

- rapid publication on acceptance

- support for research data, including large and complex data types

- gold Open Access which fosters wider collaboration and increased citations

- maximum visibility for your research: over 100M website views per year

At BMC, research is always in progress.

Learn more biomedcentral.com/submissions 